\title{
Seroprevalence of antibodies to SARS-CoV-2 and predictors of seropositivity among employees of a teaching hospital in New Delhi, India
}

\section{Pragya Sharma ${ }^{1 \oplus}$, Rohit Chawla ${ }^{2}$, Ritika Bakshi $^{1 \oplus}$, Sonal Saxena $^{2 \oplus}$, Saurav Basu ${ }^{1 \oplus}$, Pradeep Kumar Bharti ${ }^{2}$, Meera Dhuria ${ }^{3}$, S. K. Singh ${ }^{3}$, Panna Lal $^{1}{ }^{1}$}

\author{
${ }^{1}$ Department of Community Medicine, Maulana Azad Medical College, New Delhi, India \\ ${ }^{2}$ Department of Microbiology, Maulana Azad Medical College, New Delhi, India \\ ${ }^{3}$ Division of Epidemiology, National Centre for Disease Control, Delhi, India
}

Received: November 17, 2020

Revised: January 28, 2021

Accepted: February 7, 2021

Corresponding author:

Saurav Basu

Department of Community

Medicine, Maulana Azad

Medical College, 2 BSZ Marg,

New Delhi 110002, India

E-mail: saurav.basu1983@

gmail.com
Objectives: Healthcare workers (HCWs) are at a high risk of contracting severe acute respiratory syndrome coronavirus 2 (SARS-CoV-2) due to the increased likelihood of clinical exposure during patient management. The study objective was to determine the seroprevalence of antibodies to SARS-CoV-2 and its predictors among hospital employees.

Methods: The cross-sectional study was conducted at a teaching hospital from August 2020 to September 2020 among 1,401 employees, including 1,217 HCWs, in New Delhi, India. The serum samples were examined for immunoglobulin G (IgG) antibodies to SARS-CoV-2 using the COVID Kavach-Anti-SARS-CoV-2 IgG Antibody Detection enzyme-linked immunosorbent assay kit. Data were collected electronically using the EpiCollect mobile platform. A $p<0.05$ was considered to indicate statistical significance.

Results: A total of 169 participants (12.1\%) had detectable IgG antibodies to SARS-CoV-2. The highest seropositivity rate was observed in the administrative staff (20.1\%), while it was lowest among medical doctors ( $5.5 \%, p<0.001)$. Male sex and ever having lived in a containment zone were independently associated with past infection with SARS-CoV-2.

Conclusion: The seroprevalence of SARS-CoV-2 infection in health workers may be lower than in the general population in New Delhi. However, nonpharmaceutical interventions were not associated with a reduction in the risk of acquisition of SARS-CoV-2.

Keywords: Communicable diseases; COVID-19; Occupational health

\section{Introduction}

Severe acute respiratory syndrome coronavirus 2 (SARS-CoV-2) emerged as a global pandemic since its initial identification in December 2019 [1]. The clinical spectrum of coronavirus disease 2019 (COVID-19), which is caused by SARS-CoV-2, ranges from asymptomatic to severe respiratory symptoms and death, with significantly higher mortality in individuals with comorbidities and among elderly patients [2]. In India, the second-most populous country of 
the world, 6.7 million cases and 104,555 deaths attributed to COVID-19 have been recorded as of October 7, 2020. In the state of New Delhi, the Indian capital city; 295,236 cases and 5,581 deaths were observed during the same period [3].

It is well-established that healthcare workers (HCWs) comprise a population subgroup that is at a high risk of contracting SARS-CoV-2 infection due to clinical exposure during the management of suspected or confirmed COVID-19 patients [4]. The risk is further accentuated due to the incorrect use of personal protective equipment (PPE) and nonadherence to the recommended infection prevention and control guidelines [5].

The World Health Organization has estimated that 1.4 million COVID-19 infections occurred among HCWs worldwide as of August 2020 [6]. In India, according to an unofficial estimate, a total of 2,174 and 382 COVID-19 related infections and deaths, respectively, have been reported among medical doctors as of September 10, 2020, highlighting a 16-fold higher case fatality rate compared to the general population [7].

Early identification, isolation, and treatment of HCWs are indispensable tools for limiting the spread of SARS-CoV-2 among co-workers and non-COVID-19 patients within a healthcare facility. Understanding the risk factors related to the transmission of infection within healthcare settings can guide effective planning and implementation of evidencebased strategies for protecting HCWs.

Establishing the true infection rate of SARS-CoV-2 among HCWs requires an assessment of antibody seroprevalence since asymptomatic cases constitute approximately $40 \%$ to $45 \%$ of all SARS-CoV-2 infections [8]. Therefore, we conducted this study to determine the seroprevalence of antibodies to SARS-CoV-2 and its predictors among employees of a teaching hospital in New Delhi, India.

\section{Materials and Methods}

\section{Study Design and Setting}

We conducted a cross-sectional study from August 2020 to September 2020 at a teaching hospital located in New Delhi, India. The tertiary care hospital was designated as a dedicated COVID-19 hospital in April 2020, and has since treated more than 9,000 moderate to severe cases of COVID-19 [9]. No non-COVID-19 patients were admitted or treated at the hospital during the pandemic.

HCWs at the hospital deployed to the COVID-19 isolation wards currently work 15-day shifts, followed by 7 days of quarantine and another 7 days of home isolation. All HCWs working at the hospital could opt to be tested for COVID-19 if they self-reported any breach in PPE or developing symptoms suggestive of the disease. Medical personnel attending to COVID-19 patients in the wards and intensive care units were provided complete PPE kits including gowns, gloves, and N95 respirators. Administrative staff who were unlikely to come in contact with suspected COVID-19 patients were provided with surgical masks. Wearing an N95 or a surgical mask was mandatory for all hospital employees. Furthermore, employees of the hospital without any preexisting cardiac abnormalities were recommended the to receive the Indian Council of Medical Researchapproved hydroxychloroquine (HCQ) prophylaxis regimen, the intake of which was completely voluntary [10].

\section{Sampling Strategy}

Sites for immunoglobulin G (IgG) SARS-CoV-2 antibody testing were organized at different locations in the medical school and the hospital complex, and information about testing sites was disseminated through notice boards, banners, flyers, and WhatsApp groups. All the employees were invited to participate in the study. The hospital campus includes a medical college, a dental college, an eye hospital, and a tertiary care hospital with approximately 3,000 employees. Participation in this study was a one-time activity, and seronegative participants were not retested subsequently.

\section{Inclusion Criteria}

We included doctors, nurses, housekeeping staff, biomedical waste collectors, and administrative staff in the study.

\section{Methodology}

The participants were informed that seropositivity would indicate past infection. A blood sample $(3 \mathrm{~mL})$ was collected in a serum separator tube under aseptic conditions and transported to the testing facility upright in a transport box within 3 hours after collection. The serum samples were tested for qualitative detection of IgG antibodies to SARSCoV-2 using the COVID Kavach-Anti-SARS-CoV-2 Human IgG ELISA Kit (ICMR-NIV, Pune, India). The sensitivity and specificity of the assay have been reported to be $92.4 \%$ and 97.9\%, respectively [11].

\section{Data Collection}

We collected participant information on sociodemographic variables and on occupational exposure-related risk factors (Suppl. 1) through face-to-face interviews, and the data were captured using the paperless mobile EpiCollect platform [12].

\section{Operational Definitions}

Participants were characterized as high-risk if they were directly involved in the care of COVID-19 patients or handling of 
their clinical samples, and were considered low-risk otherwise. Containment zones were defined by the local administration, usually as places within a 1-km radius where 3 or more cases were detected. Within these zones, movement and access to the neighborhood were significantly restricted with exemptions for essential services staff [13].

\section{Statistical Analysis}

Data were analyzed with IBM SPSS ver. 25.0 (IBM Corp., Armonk, NY, USA). The results were expressed as frequency and proportions for categorical variables, and mean and standard deviation for continuous variables. Associations between categorical variables were assessed using the chisquare test. The variables that were significantly associated with the presence of the IgG antibody to SARS-CoV-2 were included in a binary logistic regression model. A $p<0.05$ was considered to statistical significance.

\section{Results}

\section{Sociodemographic Characteristics}

We enrolled a total of 1,401 participants, including 769 males (54.9\%) and 632 females (45.1\%). The median age of the participants was 33 years (interquartile range, 27-45 years). The participants comprised doctors (43.8\%), nursing personnel (18.1\%), auxiliary health workers $(25.1 \%)$, and administrative staff (13.1\%). A total of 466 participants (33.3\%) reported engaging in high-risk activities.

\section{Seroprevalence of IgG Antibodies to SARS-CoV-2}

A total of 169 (12.1\%; 95\% confidence interval [CI], 10.4-13.8) participants, and 132 (10.8\%; 95\% CI, 9.1-12.6) ( $n=1,217)$ HCWs (doctors and nurses), had detectable IgG antibodies against SARS-CoV-2. Among the various categories of participants, administrative staff (20.1\%) had the highest seropositivity rate, followed by auxiliary staff (18.5\%), nursing staff (13.0\%), and doctors $(5.5 \% ; p<0.001)$. The seroprevalence of SARSCoV-2 among the healthcare employees observed in this study was less than half of that observed in the general population in the state during a similar period (Figure 1).

In the bivariate analysis, the seropositivity was significantly higher in participants aged $\geq 34$ years, of the male sex, performing administrative duties, having low body mass index $\left(<18.5 \mathrm{~kg} / \mathrm{m}^{2}\right)$, with a history of COVID-19 diagnosis through real-time polymerase chain reaction (PCR) or rapid antigen test, and a history of having ever consumed any alternative medication (Table 1). Although not statistically significant, the seropositivity was higher in participants with any self-reported preexisting chronic illness $(p=0.062)$ and in those who did not undertake the HCQ prophylaxis regimen $(p=0.246)$.

Among the 101 participants with a previous history of COVID-19 infection diagnosed with real-time PCR or rapid antigen test, 42 participants (41.6\%) were IgG-seropositive. In the participants with an absence of documented COVID-19 infection ( $n=1,300), 127$ (9.76\%) were IgG-seropositive. Moreover, the proportion of health workers with seropositivity was higher in those who reported a breach of PPE during patient management compared to those who did not, but this difference did not reach statistical significance $(p=0.139)$ (Table 2).

We conducted a binary logistic regression analysis by

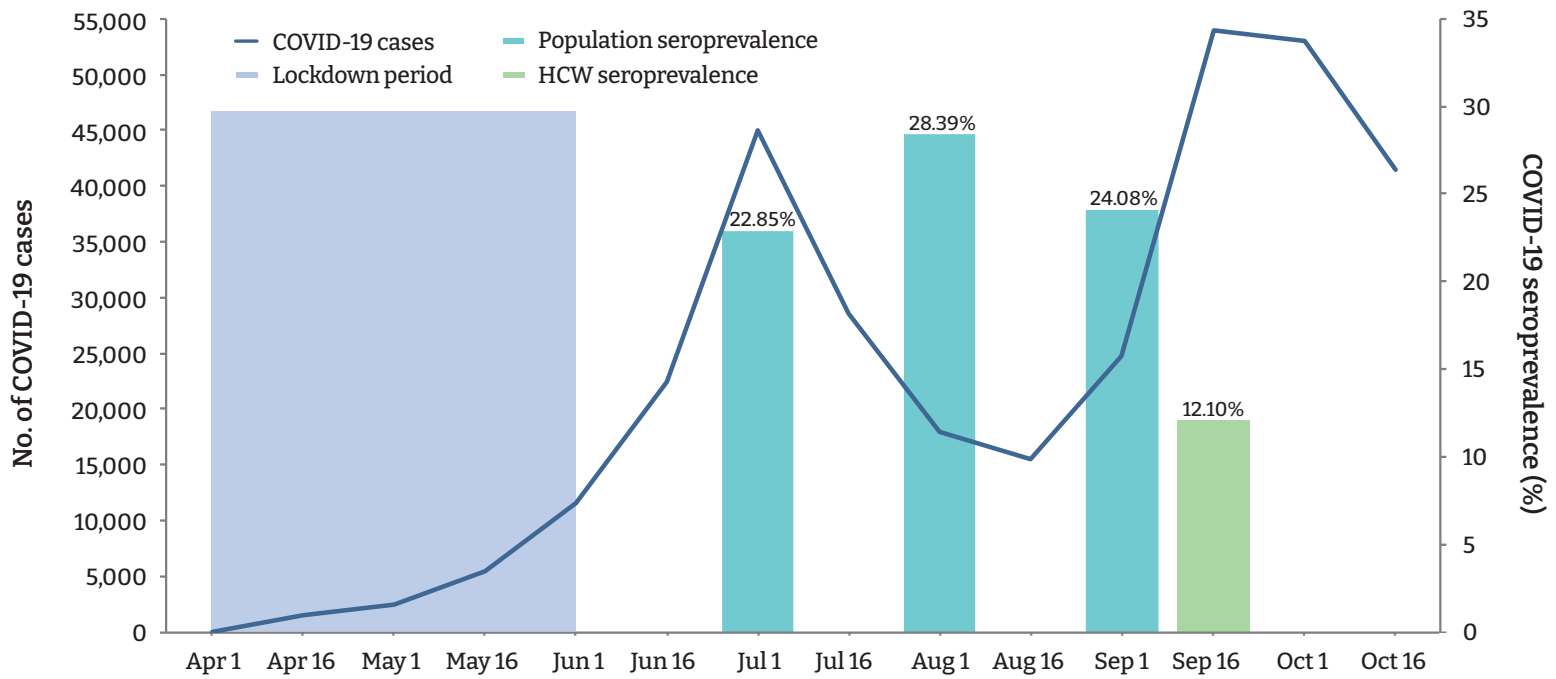

Figure 1. Epidemic curve of severe acute respiratory syndrome coronavirus 2 (SARS-CoV-2) in Delhi (April-October 2020). COVID-19, coronavirus disease 2019; HCW, healthcare worker. 
Table 1. Distribution of factors associated with IgG seropositivity to SARS-CoV-2 $(n=1,401)$

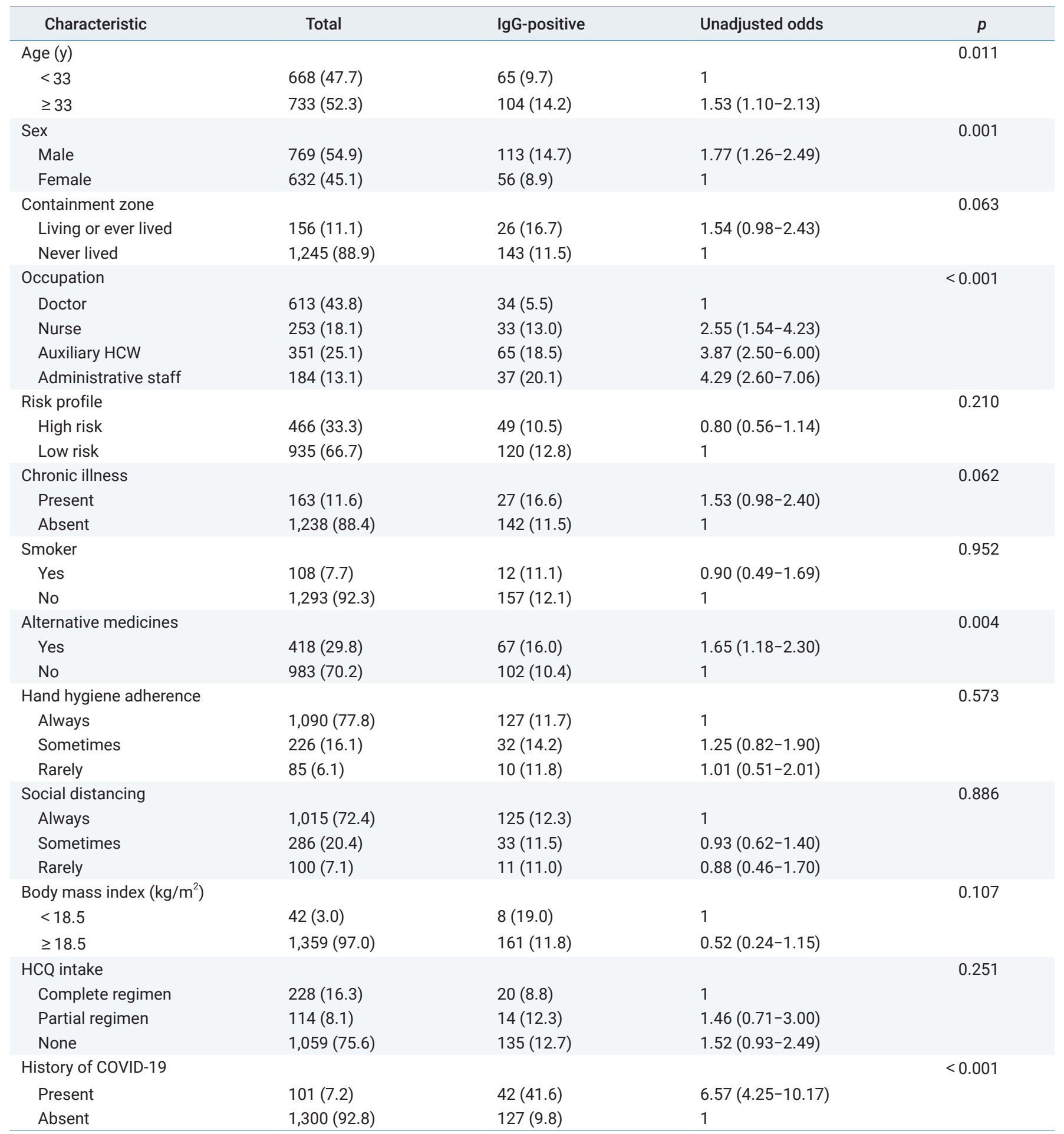

Data are presented as $n$ (\%) or odds ratio (95\% confidence interval).

IgG, immunoglobulin G; SARS-CoV-2, severe acute respiratory syndrome coronavirus 2; HCW, healthcare worker; HCQ, hydroxychloroquine; COVID-19, coronavirus disease 2019.

including variables that were statistically significant in the bivariate analysis $(p<0.05)$. The model was found to be statistically significant $\left(\chi^{2}(4)=69.76 ; p<0.001\right)$. The model correctly classified $87.9 \%$ of cases. The Hosmer-Lemeshow goodness of fit test-statistic had a $p$-value of 0.415 , from which we concluded that the model estimated the data 
Table 2. Distribution of exposure characteristics among healthcare workers and association with IgG seropositivity to SARS-CoV-2 $(n=1,217)$

\begin{tabular}{|c|c|c|c|c|}
\hline Characteristic & Total & IgG-positive & OR $(95 \% \mathrm{CI})$ & $p$ \\
\hline Direct skin contact & & & & 0.610 \\
\hline Reported & $54(4.4)$ & $7(13.0)$ & $1.24(0.55-2.80)$ & \\
\hline Not reported & $1,163(95.6)$ & $125(10.7)$ & 1 & \\
\hline Direct fluid contact & & & & 0.370 \\
\hline Reported & $17(1.4)$ & $3(17.6)$ & $1.78(0.50-6.27)$ & \\
\hline Not reported & $1,200(98.6)$ & $129(10.8)$ & 1 & \\
\hline PPE breach $(\sim 1 \mathrm{~m})$ & & & & 0.133 \\
\hline Reported & $105(8.6)$ & $16(15.2)$ & $1.54(0.88-2.72)$ & \\
\hline Not reported & $1,112(91.4)$ & $116(10.4)$ & 1 & \\
\hline PPE breach during COVID-19 care & & & & 0.930 \\
\hline Reported & $29(2.4)$ & $3(10.3)$ & $0.95(0.28-3.17)$ & \\
\hline Not reported & $1,188(97.6)$ & $129(10.8)$ & 1 & \\
\hline PPE breach when conducting an aerosol-generating procedure & & & & 0.355 \\
\hline Reported & $22(1.8)$ & $1(4.5)$ & $0.39(0.05-2.90)$ & \\
\hline Not reported & $1,195(98.2)$ & $131(11.0)$ & 1 & \\
\hline PPE breach and contact with the patient environment & & & & 0.358 \\
\hline Reported & $78(6.4)$ & $6(7.7)$ & $0.67(0.29-1.57)$ & \\
\hline Not reported & $1,139(93.6)$ & $126(11.1)$ & 1 & \\
\hline Hand hygiene after PPE removal & & & & 0.355 \\
\hline Missed & $22(1.8)$ & $1(4.5)$ & $0.39(0.05-2.90)$ & \\
\hline Not missed/uncertain & $1,195(98.2)$ & $131(11.0)$ & 1 & \\
\hline Post-duty hand hygiene & & & & 0.357 \\
\hline Always & $958(78.7)$ & $100(10.4)$ & 1 & \\
\hline Sometimes & $190(15.6)$ & $26(13.7)$ & $1.36(0.86-2.16)$ & \\
\hline Rarely & $69(5.7)$ & $6(8.7)$ & $0.82(0.34-1.94)$ & \\
\hline Post-duty social distancing & & & & 0.641 \\
\hline Always & $893(73.4)$ & $101(11.3)$ & 1 & \\
\hline Sometimes & $241(19.8)$ & $24(10.0)$ & $0.87(0.54-1.39)$ & \\
\hline Rarely & $83(6.8)$ & $7(8.4)$ & $0.72(0.32-1.61)$ & \\
\hline
\end{tabular}

Data are presented as $n(\%)$ or OR $(95 \% \mathrm{Cl})$. Contact with body fluids (urine, blood, saliva) of a confirmed COVID-19 patient. Within a 1-m distance of a confirmed COVID-19 patient who was not wearing a mask/respirator.

IgG, immunoglobulin G; SARS-CoV-2, severe acute respiratory syndrome coronavirus 2; OR, odds ratio; Cl, confidence interval; PPE, personal protective equipment; COVID-19, coronavirus disease 2019.

acceptably. Among the variables that were found to be statistically significant in the bivariate analysis, only male sex and the type of occupation were found to be independently associated with seropositivity in the logistic regression analysis (Table 3). The administrative staff had 3.8-fold higher odds of being IgG-seropositive than the doctors.

\section{Discussion}

The present study conducted among employees of a teaching hospital in New Delhi observed that the IgG antibody to SARSCoV-2 was present in $12.1 \%$ of participants, indicating past infection with the virus. A large community-based repeated serosurvey conducted in Delhi during the same period reported detectable IgG antibodies in $24.08 \%$ to $28.39 \%$ of the population [14]. Surprisingly, in our study, the seropositivity was significantly higher among administrative staff who had a considerably lower risk of exposure to the virus than HCWs. These findings suggest that the likelihood of exposure to SARS-CoV-2 was high in the community. Furthermore, although the administrative staff were provided with facemasks and instructed to adhere to hand hygiene and social distancing measures, a lack of effective training in correctly using PPE may have disproportionately accentuated their risk compared to the HCWs.

Previous studies in India have shown variable rates of seropositivity among HCWs ranging from $2.5 \%$ in the Srinagar district [15] to $16 \%$ in Southern Rajasthan, with most cases being asymptomatic [16]. In Europe, various Italian studies have reported COVID-19 IgG seroprevalence 
Table 3. Logistic regression analysis of factors associated with IgG seropositivity to SARS-CoV-2

\begin{tabular}{llc}
\hline \multicolumn{1}{c}{ Characteristic } & Adjusted OR $(95 \% \mathrm{Cl})$ & \multicolumn{1}{c}{$p$} \\
\hline $\begin{array}{l}\text { Age }(\mathrm{y}) \\
<33\end{array}$ & 0.549 \\
$\geq 33$ & $1.11(0.79-1.57)$ & \\
Sex & & \\
$\quad$ Male & $1.45(1.00-2.10)$ & \\
$\quad$ Female & 1 (reference) & \\
Occupation & & \\
$\quad$ Doctor & 1 (reference) & $<0.001$ \\
Nurse & $2.59(1.54-4.35)$ & \\
Auxiliary & $3.34(2.09-5.31)$ & \\
Administrative staff & $3.77(2.25-6.31)$ & \\
Body mass index $\left(\mathrm{kg} / \mathrm{m}^{2}\right)$ & \\
$\quad<18.5$ & 1 (reference) & \\
$\geq 18.5$ & $0.60(0.27-1.37)$ & \\
\hline
\end{tabular}

IgG, immunoglobulin G; SARS-CoV-2, severe acute respiratory syndrome coronavirus 2; $\mathrm{OR}$, odds ratio; $\mathrm{Cl}$, confidence interval.

among hospital personnel to range from 5.1\% to 7.4\% [17,18]. In Denmark, the seropositivity among HCWs was $4.04 \%$, with a significant association observed with a history of exposure to COVID-19 patients [19]. Another study in the USA reported $6 \%$ of HCWs within a multistate hospital network had detectable SARS-CoV-2 antibodies, and within the seropositive subgroup, $69 \%$ had no prior microbiological confirmation of COVID-19 disease [20]. However, a study among a large cohort of HCWs in New York City (NYC) who were voluntarily tested reported a higher seroprevalence of antibodies (13.7\%), which was correlated with the high COVID-19 burden [21].

Similar to the present study, men were reported to be at a higher risk of SARS-CoV-2 infection in the studies conducted in Milan and Denmark [17,19]. Age was not found to be a significant predictor of seropositivity among HCWs in the NYC study, which corroborates the findings of this study [20]. Acquisition of SARS-CoV-2 infection locally from the community as the dominant mode of transmission among HCWs was also previously reported in a Dutch study [22].

In the present study, the intake of an HCQ prophylaxis regimen did not have any protective effect on the risk of contracting the SARS-CoV-2 infection. Although some early observational studies indicated protective efficacy of HCQ against COVID-19, evidence generated from later clinical trials showed that daily HCQ does not prevent SARS-CoV-2 infection [23-25].

In this study, the use of immunity boosters based on alternative systems of medicine, such as indigenous systems of Indian medicine and homeopathy, was associated with a significantly higher risk of seropositivity. The findings suggest that while immunity boosters probably offered no protection against the acquisition of SARS-CoV-2 infection, they might instill a false sense of security leading to reduced adherence to preventive measures, with increased risk of infection. Nonetheless, the potential impact of alternative systems of medicine in the management of COVID-19, including disease progression and severity, needs further evaluation.

The strengths of the study include a large sample size and collection of data through face-to-face interviews with the employees of the largest state-run teaching hospital in Northern India catering only to COVID-19 patients. However, the study has several limitations. These include the use of a cross-sectional analysis, which precluded the assessment of any temporal or causal relationships, especially changes in seroprevalence with the progression of the pandemic, which would require a prospective study design. The study was conducted in a single institutional setting, limiting the generalizability of the findings. Furthermore, the employees who did not participate in the study may have differed in their sociodemographic and clinical characteristics, leading to potential selection bias. Finally, the data on adherence to preventive measures against COVID-19 were self-reported, which may have led to recall bias.

In conclusion, the present study conducted at a teaching hospital in New Delhi observed that nearly 1 in 10 HCWs without any prior microbiological confirmation had detectable IgG antibodies to SARS-CoV-2. Considering that the doctors had the lowest seropositivity for SARS-CoV-2, whereas administrative staff had the highest seropositivity, the probability of HCW-to-HCW or patient-to-HCW transmission may be lower than the risk of transmission from other sources including the general population. However, nonpharmaceutical interventions were not associated with a reduction in the risk of acquisition of SARS-CoV-2, implying the necessity to improve training techniques among all hospital employees for maintaining the correct use of PPE on all occasions involving the risk of contracting the infection.

\section{Supplementary Material}

Table S1. All indicators except public transportation. Supplementary data is available at https://doi.org/10.24171/j.phrp.2021.12.2.06.

\section{Notes}

\section{Ethics Approval}

The study protocol was approved by the Institutional Ethics Committee (F.1/IEC/MAMC/(77/05/2020/No 202). Written and informed consent was obtained from all the participants. 


\section{Conflicts of Interest}

The authors have no conflicts of interest to declare.

\section{Funding}

None.

\section{Availability of Data}

The anonymized dataset that support the findings of this study will be made available by the corresponding author upon reasonable request.

\section{Authors' Contributions}

Conceptualization: PS, RC, RB, SS, PL, MD, SKB; Data curation: PS, RC, RB, PKB; Formal analysis: SB; Funding acquisition: Nil; Investigation: PS, RC, RB, PKB; Methodology PS, RC, RB, SB, MD; Project administration: PS, RC, PKB; Resources: PS, RC, SS, MD, SKS; Supervision: PS, SS, PL; Validation: PS, SS, MD; Visualization: SB; Writing-original draft: SB; Writing-review \& editing: all authors.

\section{Additional Contributions}

Mr. Kumar Dushyant (research associate) contributed to creating the figure for the manuscript.

\section{References}

1. Zhu N, Zhang D, Wang W, et al. A novel coronavirus from patients with pneumonia in China, 2019. N Engl J Med 2020;382:727-33.

2. Fauci AS, Lane HC, Redfield RR. Covid-19: navigating the uncharted. N Engl J Med 2020;382:1268-9.

3. Ministry of Health and Family Welfare. Government of India. 2020 [Internet]. [cited 2020 Oct 8]. Available from: https://www.mohfw. gov.in/.

4. Suarez-Garcia I, Martinez de Aramayona Lopez MJ, Saez Vicente A, et al. SARS-CoV-2 infection among healthcare workers in a hospital in Madrid, Spain. J Hosp Infect 2020;106:357-63.

5. Public Health England. COVID-19: guidance for maintaining services within health and care settings. Infection prevention and control recommendations [Internet]. Public Health England; 2020 [cited 2020 Oct 7]. Available from: https://assets.publishing.service.gov.uk/ government/uploads/system/uploads/attachment_data/file/881489/ COVID-19_Infection_prevention_and_control_guidance_complete. pdf.

6. News: Coronavirus latest: WHO says health workers account for 10\% of global infections. Deutsche Welle (DW) [Internet]. 2020 Aug 17 [cited 2020 Oct 7]. Available from: https://www.dw.com/en/ coronavirus-latest-who-says-health-workers-account-for-10-ofglobal-infections/a-54208221.

7. Kapoor A, Kapoor KM. Covid-19 related deaths among doctors in India [Preprint]. Posted 2020 Sep 30. medRxiv 2020.09.28.20202796. https:// doi.org/10.1101/2020.09.28.20202796.

8. Oran DP, Topol EJ. Prevalence of asymptomatic SARS-CoV-2 infection: a narrative review. Ann Intern Med 2020;173:362-7.

9. Dwivedi S. Delhi hospital treats 8,000 Covid patients: highest by state-run facility. The Times of India [Internet]. 2020 Oct 4 [cited 2020 Oct 7]. Available from: https://www.ndtv.com/delhi-news/delhilnjp-hospital-treats-8-000-covid-patients-highest-by-any-state- run-facility-in-india-2305037.

10. Indian Council of Medical Research. Revised advisory on the use of hydroxychloroquine (HCQ) as prophylaxis for SARS-CoV-2 infection (in supersession of previous advisory dated 23rd March, 2020) [Internet]. 2020 [cited 2020 Oct 7]. Available from: https://www.icmr. gov.in/pdf/covid/techdoc/V5_Revised_advisory_on_the_use_of_ HCQ_SARS_CoV2_infection.pdf.

11. Sapkal G, Shete-Aich A, Jain R, et al. Development of indigenous IgG ELISA for the detection of anti-SARS-CoV-2 IgG. Indian J Med Res 2020;151:444-9.

12. Aanensen DM, Huntley DM, Feil EJ, et al. EpiCollect: linking smartphones to web applications for epidemiology, ecology and community data collection. PLoS One 2009;4:e6968.

13. Ministry of Health and Family Welfare, India. Containment plan: novel coronavirus disease 2019 (COVID 19) version 2 (updated 16.05.2020) [Internet]. New Delhi: Ministry of Health and Family Welfare, India; 2020 [cited 2020 Oct 7]. Available from: https://www. mohfw.gov.in/pdf/Containmentplan16052020.pdf.

14. Sharma N, Sharma P, Basu S, et al. The seroprevalence and trends of SARS-CoV-2 in Delhi, India: a repeated population-based seroepidemiological study [Priprint]. Posted 2020 Dec 14. medRxiv 2020.12.13.20248123. https://doi.org/10.1101/2020.12.13.20248123.

15. Khan SM, Qurieshi MA, Haq I, et al. Seroprevalence of SARS-CoV-2 specific IgG antibodies in District Srinagar, northern India: a cross-sectional study. PLoS One 2020;15:e0239303.

16. Dave M, Vijayvargiya R, Poswal L, et al. Study of COVID-19 seroprevalence among healthcare workers at dedicated COVID hospital in southern Rajasthan. Indian J Clin Pract 2020;31:107-11.

17. Amendola A, Tanzi E, Folgori L, et al. Low seroprevalence of SARSCoV-2 infection among healthcare workers of the largest children hospital in Milan during the pandemic wave. Infect Control Hosp Epidemiol 2020;41:1468-9.

18. Sotgiu G, Barassi A, Miozzo M, et al. SARS-CoV-2 specific serological pattern in healthcare workers of an Italian COVID-19 forefront hospital. BMC Pulm Med 2020;20:203.

19. Iversen K, Bundgaard H, Hasselbalch RB, et al. Risk of COVID-19 in health-care workers in Denmark: an observational cohort study. Lancet Infect Dis 2020;20:1401-8.

20. Self WH, Tenforde MW, Stubblefield WB, et al. Seroprevalence of SARS-CoV-2 among frontline health care personnel in a multistate hospital network: 13 academic medical centers, April-June 2020. MMWR Morb Mortal Wkly Rep 2020;69:1221-6.

21. Moscola J, Sembajwe G, Jarrett M, et al. Prevalence of SARS-CoV-2 antibodies in health care personnel in the New York City area. JAMA 2020;324:893-5.

22. Kluytmans-van den Bergh MF, Buiting AG, Pas SD, et al. Prevalence and clinical presentation of health care workers with symptoms of coronavirus disease 2019 in 2 Dutch hospitals during an early phase of the pandemic. JAMA Netw Open 2020;3:e209673.

23. Chatterjee P, Anand T, Singh KJ, et al. Healthcare workers \& SARS- 
CoV-2 infection in India: a case-control investigation in the time of COVID-19. Indian J Med Res 2020;151:459-67.

24. Abella BS, Jolkovsky EL, Biney BT, et al. Efficacy and safety of hydroxychloroquine vs placebo for pre-exposure SARS-CoV-2 prophylaxis among health care workers: a randomized clinical trial. JAMA Intern Med 2021;181:195-202.
25. Boulware DR, Pullen MF, Bangdiwala AS, et al. A randomized trial of hydroxychloroquine as postexposure prophylaxis for Covid-19. N Engl J Med 2020;383:517-25. 